\title{
SELF-ADJOINTNESS OF THE MOMENTUM OPERATOR WITH A SINGULAR TERM
}

\author{
MICHIAKI WATANABE AND SHUJI WATANABE
}

(Communicated by Palle E. T. Jorgensen)

\begin{abstract}
Self-adjointness is shown of the momentum operator $p=-i(d / d x)$ $+i(c / x) R, R u(x)=u(-x)$, with domain $\left\{u \in H^{1}\left(\mathbf{R}^{1}\right): u / x \in L^{2}\left(\mathbf{R}^{1}\right)\right\}$ when $c>1$ or $c<-1$. This operator appears in a harmonic oscillator system with the generalized commutation relations by Wigner: $i p=[x, H]$ and $-i x=[p, H]$ for the Hamiltonian $H$ and the multiplication operator $x$.

The proof is carried out by generation of a unitary group in terms of ip, based on the Hille-Yosida theorem and Stone's theorem. The result is applied to the self-adjoitness of $H=\left(p^{2}+x^{2}\right) / 2$.
\end{abstract}

\section{INTRODUCTION AND THE MAIN RESULT}

This paper concerns the self-adjointness of the operator:

$$
p=-i(d / d x)+i(c / x) R, \quad R u(x)=u(-x),
$$

with a real constant $c$ specified later. This operator, called the momentum operator, was expressed in this form by Yang [6] to deal with a one-dimensional harmonic oscillator system governed by the generalized commutation relations, due to Wigner:

$$
i p=[x, H] \text { and }-i x=[p, H] .
$$

Here the Hamiltonian is of the classical form $H=\left(p^{2}+x^{2}\right) / 2$, and $x$ is assumed to be the multiplication by $x$.

In the case of $c=0, p$ is a well-known example of a self-adjoint operator in $L^{2}\left(\mathbf{R}^{1}\right)$ (see e.g. Yosida [7, p. 198]). The purpose of this paper is to prove the following theorem, and then to apply it to the self-adjointness of the Hamiltonian $H$ :

Theorem 1. If $|c|>1$, then the operator $p$ is self-adjoint on the set

$$
V=\left\{u \in H^{1}\left(\mathbf{R}^{1}\right): u / x \in L^{2}\left(\mathbf{R}^{1}\right)\right\} .
$$

Received by the editors January 30, 1989.

1980 Mathematics Subject Classification (1985 Revision). Primary 47B25, 47D45; Secondary $81 \mathrm{C} 10,81 \mathrm{C} 12$.

Key words and phrases. Self-adjointness, generation of unitary group, perturbation, momentum operator, Hamiltonain. 
To begin with we study fundamental properties of $p$. A simple calculation shows that $p$ is symmetric in $\left(L^{2}\left(\mathbf{R}^{1}\right),(\cdot, \cdot)\right)$. It is easy to verify that $C_{0}^{\infty}(Q)$ with $Q=\mathbf{R}^{1}-\{0\}$ is dense in $L^{2}\left(\mathbf{R}^{1}\right)$ and hence so is $V$.

Lemma 1. The following hold for $A=i p$ :

(i) The domain $D(A)(=V)$ of $A$ is dense in $L^{2}\left(\mathbf{R}^{1}\right)$;

(ii) $\operatorname{Re}(A u, u)=0$ for $u \in D(A)$.

In the following, we shall show with the aid of the Hille-Yosida theorem that $A$ generates a strongly continuous unitary group on $L^{2}\left(\mathbf{R}^{1}\right)$ to obtain the selfadjointness of $p(=-i A)$ by Stone's theorem. To examine the range $R(\lambda-A)$ of $\lambda-A$, we deal with the equation

$$
\lambda u-D u+(c / x) R u=f, \quad D=d / d x,
$$

for given $\lambda \in Q$ and $f \in L^{2}\left(\mathbf{R}^{1}\right)$. If there exist an even function $u_{1}$ and an odd function $u_{2}$ satisfying

$$
\left\{\begin{array}{l}
\lambda u_{1}-(D+c / x) u_{2}=(f+R f) / 2, \\
\lambda u_{2}-(D-c / x) u_{1}=(f-R f) / 2
\end{array}\right.
$$

then $u_{1}+u_{2}$ is a solution of (1) since $u_{1}-u_{2}=R\left(u_{1}+u_{2}\right)$. Moreover it is expected that for $u_{1}$ and $u_{2}$

$$
\begin{aligned}
& \lambda^{2} u_{1}-(D+c / x)(D-c / x) u_{1}=\lambda \bar{f}+(D+c / x) \underline{f}, \\
& \lambda^{2} u_{2}-(D-c / x)(D+c / x) u_{2}=\lambda \underline{f}+(D-c / x) \bar{f}
\end{aligned}
$$

hold true in some weak sense, where

$$
\bar{f}=(f+R f) / 2 \text { and } \underline{f}=(f-R f) / 2 .
$$

Thus our main task is to look for such functions as $u_{1}$ and $u_{2}$ in the above.

\section{Self-ADJOINTNESS OF THE OPERATOR $p$}

Let us consider the symmetric sesquilinear forms on $V \times V$ :

$$
\begin{aligned}
& b_{1}(u, v)=\lambda^{2}(u, v)+(D u, D v)+\left(c^{2}-c\right)(u / x, v / x), \\
& b_{2}(u, v)=\lambda^{2}(u, v)+(D u, D v)+\left(c^{2}+c\right)(u / x, v / x) .
\end{aligned}
$$

If $|c|>1, V$ becomes a Hilbert space with each of the inner products $b_{1}(\cdot, \cdot)$ and $b_{2}(\cdot, \cdot)$ for a fixed $\lambda \in Q$.

Let $V_{0}$ be the closure in $V$ of $C_{0}^{\infty}(Q)$. Then we have

$$
\begin{aligned}
& b_{1}(u, v)=\lambda^{2}(u, v)+((D-c / x) u,(D-c / x) v), \\
& b_{2}(u, v)=\lambda^{2}(u, v)+((D+c / x) u,(D+c / x) v)
\end{aligned}
$$

for $u, v \in C_{0}^{\infty}(Q)$ and hence for $u, v \in V_{0}$. Using Riesz' representation theorem, we have that for given $\lambda \in Q$ and $f \in L^{2}\left(\mathbf{R}^{1}\right)$ with $\bar{f}$ and $\underline{f}$ defined 
by (3), there exist unique $u_{1}$ and $u_{2}$ in $V_{0}$ satisfying, for all $\phi, \psi \in V_{0}$,

$$
\left\{\begin{array}{l}
b_{1}\left(u_{1}, \phi\right)=\lambda(\bar{f}, \phi)-(\underline{f},(D-c / x) \phi), \\
b_{2}\left(u_{2}, \psi\right)=\lambda(\underline{f}, \psi)-(\bar{f},(D+c / x) \psi) .
\end{array}\right.
$$

Here we have used that $\lambda(\bar{f}, \cdot)-(\underline{f},(D-c / x) \cdot)$ and $\lambda(\underline{f}, \cdot)-(\bar{f},(D+c / x) \cdot)$ are continuous conjugate linear functionals on $V_{0}$.

Let us here examine the properties of $u_{1}$ and $u_{2}$ to prove the following:

Lemma 2. If $|c|>1$, then we have

$$
R(\lambda-A)=L^{2}\left(\mathbf{R}^{1}\right) \quad \text { for } \lambda \in Q .
$$

Proof. The proof is divided into three steps:

Step 1. First we shall show that $u_{1}$ is even and $u_{2}$ is odd. In view of $R D=-D R$ and $R(c / x)=-(c / x) R$, we see

$$
\begin{aligned}
b_{1}\left(R u_{1}, \phi\right) & =b_{1}\left(u_{1}, R \phi\right) \\
& =\lambda(\bar{f}, \phi)-(\underline{f},(D-c / x) \phi), \\
b_{2}\left(-R u_{2}, \psi\right) & =-b_{2}\left(u_{2}, R \psi\right) \\
& =\lambda(\underline{f}, \psi)-(\bar{f},(D+c / x) \psi)
\end{aligned}
$$

for all $\phi, \psi \in V_{0}$. Thus the uniqueness of $u_{1}$ and $u_{2}$ gives

$$
R u_{1}=u_{1} \text { and } R u_{2}=-u_{2} \text {. }
$$

Step 2. Next we will show that $v_{1}$ and $v_{2}$ defined by

$$
\left\{\begin{array}{l}
v_{1}=\lambda^{-1}\left(\bar{f}+(D+c / x) u_{2}\right), \\
v_{2}=\lambda^{-1}\left(\underline{f}+(D-c / x) u_{1}\right)
\end{array}\right.
$$

satisfy, for all $\phi \in C_{0}^{\infty}(Q)$,

$$
\left\{\begin{array}{l}
\left(u_{1}-v_{1}, \lambda^{2} \phi+L\left(c^{2}-c\right) \phi\right)=0 \\
\left(u_{2}-v_{2}, \lambda^{2} \phi+L\left(c^{2}+c\right) \phi\right)=0
\end{array}\right.
$$

where $L(k)=-D^{2}+k / x^{2}$ for a real constant $k$.

Indeed, substituting (5) into (4), we have

$$
\begin{aligned}
& \lambda\left(u_{1}, \phi\right)+\left(v_{2},(D-c / x) \phi\right)=(\bar{f}, \phi), \\
& \lambda\left(u_{2}, \psi\right)+\left(v_{1},(D+c / x) \psi\right)=(\underline{f}, \psi) .
\end{aligned}
$$

On the other hand, we obtain directly from (5)

$$
\begin{aligned}
& \lambda\left(v_{1}, \phi\right)+\left(u_{2},(D-c / x) \phi\right)=(\bar{f}, \phi), \\
& \lambda\left(v_{2}, \psi\right)+\left(u_{1},(D+c / x) \psi\right)=(\underline{f}, \psi)
\end{aligned}
$$

by integration by parts. Thus we find

$$
\left\{\begin{array}{l}
\lambda\left(u_{1}-v_{1}, \phi\right)=\left(u_{2}-v_{2},(D-c / x) \phi\right), \\
\lambda\left(u_{2}-v_{2}, \psi\right)=\left(u_{1}-v_{1},(D+c / x) \psi\right)
\end{array}\right.
$$

for all $\phi, \psi \in V_{0}$, from which (6) follows. 
Step 3. Finally we prove that $v_{1}=u_{1}$ and $v_{2}=u_{2}$, that is, $u_{1}$ and $u_{2}$ satisfy (2).

In the case of $c>1$, since $c^{2}+c>2$, the following lemma is applicable to our oprator $L\left(c^{2}+c\right)$ :

Lemma 3. If $k \geq 3 / 4$, then $L(k)=-D^{2}+k / x^{2}$ is essentially self-adjoint on $C_{0}^{\infty}(Q)$

For the proof, see e.g. Kato [2, pp. 402-403] or Okazawa [4, pp. 697-698]. From this lemma we see that the adjoint operator $L^{*}(k)$ of $L(k)$ for $k \geq 3 / 4$ coincides with the smallest closed extension of $L(k)$, and satisfies

$$
R\left(\lambda^{2}+L(k)^{*}\right)=L^{2}\left(\mathbf{R}^{1}\right) \quad \text { for } \lambda \in Q .
$$

Let us continue the proof of Lemma 2, Step 3. Applying (8) with $k=c^{2}+c$ to the second equality of (6), we obtain $v_{2}=u_{2}$. Hence the first of (7) becomes $\left(u_{1}-v_{1}, \phi\right)=0$ for all $\phi \in V_{0}$. Since $V_{0}$ is dense in $L^{2}\left(\mathbf{R}^{1}\right)$, we obtain $v_{1}=u_{1}$.

In the case of $c<-1$, since $c^{2}-c>2$, a similar argument by the first of (6), the second of (7), and (8) with $k=c^{2}-c$ also gives $v_{1}=u_{1}$ and then $v_{2}=u_{2}$. Q.E.D.

Thus we have proved Theorem 1. In fact, as long as $|c|>1,-i A(=p)$ is self-adjoint if and only if Lemmas 1 and 2 are true. See e.g. Goldstein [1, p. 32].

\section{Application to the Hamiltonian $H$}

Let us consider the operator $H$ given by

$$
H=\left(p^{2}+x^{2}\right) / 2 .
$$

We have shown in the above the self-adjointness of $p$ and hence of $p^{2}$ with domain $D\left(p^{2}\right)=\{u \in V: p u \in V\}$ under the restriction $|c|>1$. Clearly $x^{2}$ is self-adjoint on $D\left(x^{2}\right)=\left\{u \in L^{2}\left(\mathbf{R}^{1}\right): x^{2} u \in L^{2}\left(\mathbf{R}^{1}\right)\right\}$.

As mentioned in the introduction, we shall discuss the self-adjointness of $H$ as an application of the result for $p$, and then as a consequence of a similar argument for $i H$. In this section Okazawa's theory [4] plays an important role.

The following is a corollary of Theorem 1 :

Theorem 2. If $|c|>1$, then $H$ is self-adjoint on

$$
W_{1}=\left\{u \in D\left(p^{2}\right): x^{2} u \in L^{2}\left(\mathbf{R}^{1}\right)\right\} .
$$

Proof. We will apply Okazawa's perturbation theorem [4, Theorem 5.4] to the nonnegtive self-adjoint operators $p^{2}$ and $x^{2}$. An elementary calculation gives

$$
\operatorname{Re}\left(p^{2} u, n x^{2}\left(n+x^{2}\right)^{-1} u\right) \geq-(u, u)
$$

for $u \in D\left(p^{2}\right)$ and $n=1,2, \ldots$, where $n x^{2}\left(n+x^{2}\right)^{-1}$ is the Yosida-approximation of $x^{2}$. Indeed, the left-hand side equals

$$
\left(p u, n x^{2}\left(n+x^{2}\right)^{-1} p u\right)+\operatorname{Im}\left(p u, 2 n^{2} x\left(n+x^{2}\right)^{-2} u\right) .
$$


Thus $H$ is self-adjoint on $W_{1}\left(=D\left(p^{2}\right) \cap D\left(x^{2}\right)\right)$. Q.E.D.

It is not very easy to deal with the domain $W_{1}$, which is indeed a subset of the abstract set $D\left(p^{2}\right)$. Let us observe here that

$$
p^{2} u=-D^{2} u+\left(c / x^{2}\right)(c-R) u
$$

holds for all $u \in C_{0}^{\infty}(Q)$. Therefore we may use the same notation $H$ to denote the operator

$$
H=\left\{-D^{2}+\left(c / x^{2}\right)(c-R)+x^{2}\right\} / 2 .
$$

Recalling the technique used for ip in the preceding sections, we can establish the self-adjointness of this operator $H$ on the simpler domain

$$
W_{2}=\left\{u \in H^{2}\left(\mathbf{R}^{1}\right): u / x^{2}, x^{2} u \in L^{2}\left(\mathbf{R}^{1}\right)\right\},
$$

however, under somewhat stronger condition on $c$ :

Theorem 3. If $|c|>3 / 2$, then $H$ is self-adjoint on $W_{2}$.

Proof. An easy calculation shows that Lemma 1 holds true with $A=i H$. To prove Lemma 2 with $A=i H$, we will look for $w_{1}$ and $w_{2}$ in $W_{2}$ satisfying (cf. (2))

$$
\left\{\begin{array}{l}
\lambda w_{1}-i\left\{L\left(c^{2}-c\right)+x^{2}\right\} w_{1}=\bar{f}, \\
\lambda w_{2}-i\left\{L\left(c^{2}+c\right)+x^{2}\right\} w_{2}=\underline{f},
\end{array}\right.
$$

respectively, for given $\lambda \in Q$ and $f \in L^{2}\left(\mathbf{R}^{1}\right)$.

Since $|c|>3 / 2$, both $L\left(c^{2}+c\right)$ and $L\left(c^{2}-c\right)$ are self-adjoint on the set

$$
D(L)=\left\{u \in H^{2}\left(\mathbf{R}^{1}\right): u / x^{2} \in L^{2}\left(\mathbf{R}^{1}\right)\right\}
$$

by the following lemma due to Okazawa [4, Theorem 6.8]:

Lemma 4. If $k>3 / 4$, then $L(k)=-D^{2}+k / x^{2}$ is self-adjoint on $D(L)$.

Let us turn to the proof of Theorem 3. Noting

$$
\begin{aligned}
& \left(L(k) u, n x^{2}\left(n+x^{2}\right)^{-1} u\right) \\
& \quad=\left(D u, n x^{2}\left(n+x^{2}\right)^{-1} D u\right)+\left(D u, 2 n^{2} x\left(n+x^{2}\right)^{-2} u\right)+\left(k u, n\left(n+x^{2}\right)^{-1} u\right),
\end{aligned}
$$

we obtain the estimate similar to (9):

$$
\operatorname{Re}\left(L(k) u, n x^{2}\left(n+x^{2}\right)^{-1} u\right) \geq-(u, u)
$$

for $u \in D(L)$ and $n=1,2, \ldots$. Hence Okazawa's perturbation theorem again gives the self-adjointness on $W_{2}\left(=D(L) \cap D\left(x^{2}\right)\right)$ of $L(k)+x^{2}$ with $k>3 / 4$. The operators $L\left(c^{2}+c\right)+x^{2}$ and $L\left(c^{2}-c\right)+x^{2}$ are now self-adjoint and, by Stone's theorem, there exist unique $w_{1}$ and $w_{2}$ in $W_{2}$ satisfying (10). Evidently $w_{1}$ is even and $w_{2}$ is odd, and $w_{1}+w_{2}$ is a solution of

$$
\lambda w-2 i H w=f
$$


for every $\lambda \in Q$ and $f \in L^{2}\left(\mathbf{R}^{1}\right)$. Therefore, as long as $|c|>3 / 2, R(\lambda-i H)$ equals $L^{2}\left(\mathbf{R}^{1}\right)$ for $\lambda \in Q$.

Thus we have proved Lemmas 1 and 2 with $A=i H$ and $|c|>3 / 2$, and equivalently Theorem 3. Q.E.D.

As for the Hamiltonian $H$ together with the momentum operator $p$, we refer the reader to the recent paper [8].

\section{REFERENCES}

1. J. A. Goldstein, Semigroups of linear operators and applications, Oxford University Press, New York, 1985/ Clarendon Press, Oxford, 1985.

2. T. Kato, Perturbation theory for linear operators, Springer-Verlag, Berlin and New York, 1966.

3. Y. Ohnuki and S. Kamefuchi, Quantum field theory and parastatistics, University of Tokyo Press, Tokyo, 1982/ Springer-Verlag, Berlin, 1982.

4. N. Okazawa, On the perturbation of linear operators in Banach and Hilbert spaces, J. Math. Soc. Japan 34 (1982), 677-701.

5. M. Reed and B. Simon, Methods of modern mathematical physics, II, Fourier analysis, selfadjointness, Academic Press, New York, 1975.

6. L. M. Yang, Phys. Rev. 84 (1951), 788.

7. K. Yosida, Functional analysis, 6th ed., Springer-Verlag, Berlin, Heidelberg and New York, 1980.

8. S. Watanabe, A harmonic oscillator system with the generalized commutation relations, J. Math. Phys., 30 (1989), 376-379.

Faculty of General Education, Nigata University, Nilgata, 950-21 Japan

Department of Physics, Nagoya University, Nagoya, 464 Japan 